City University of New York (CUNY) CUNY Academic Works

1968

\title{
Analysis of Time Usage in Bell System Business Offices
}

William (Bill) H. Williams

CUNY Hunter College

Hwei Chen

Bell Labs

\section{How does access to this work benefit you? Let us know!}

More information about this work at: https://academicworks.cuny.edu/hc_pubs/646

Discover additional works at: https://academicworks.cuny.edu

This work is made publicly available by the City University of New York (CUNY).

Contact: AcademicWorks@cuny.edu 
Copyright $\odot 1969$ American Telephone and Telegraph Company

Reprinted from The Bell System Technical Journal.

Vol. 48, No. 7, September, 1969

Printed in U.S.A.

\title{
An Analysis of Time Usage in Bell System Business Offices
}

\author{
By W. H. WILLTAMS and H. CHEN \\ (Manuscript received November 4, 1968)
}

The everyday contact with customers of the Bell System is carried out in approximately 2100 business offices. The assessment of such a large number of offices makes the continued improvement of formal office measurement schemes aliractive. This paper describes an analysis of models for time usage in Bell System business offices. In addition, it was hoped that these models would be potentially useful for interoffice comparisons. A model for single offices is described first. This is followed by the development of a multioffice model which is constructed in such a way that it has good statistical characteristics and attempts to make the office comparisons as fair as possible.

The inputs to the multioffice model are: (i) the gross time used by each business office, (ii) the number of contacts that each office had with business and residence customers, (iii) the number of accounts carried by each office, and (iv) certain characteristics which were judged to reflect the nature of the exogenous demand put on the office, for example, percent of business main telephones.

I. TIME MEASUREMENT SCHEMES AND THE BELL SYSTEM BUSINISS OFFICES

The everyday contact with customers of the Bell System is carried out in approximately 2100 business offices. These offices have many functions. To specify just a few, most orders for telephone service, toll inquiries, and complaints of various kinds are handled by them. Consequently these offices are very important and need to be well run. However, their large number emphasizes the need for formal office measurement schemes which can be studied objectively.

While such schemes can be very useful, they can also contain very troublesome features. The first of these troubles relates to the operational definition of the word efficiency. It should not be so broad as to be meaningless or misleading, nor should it be overly narrow. The re- 
sult of a narrow definition of efficiency is likely to be multiple measures which would be very unwieldy with a large number of offices. Additionally, this definition must reflect the internal and external office characteristics that are statistically associated with efficiency.

While the problems of meaning and measurement are obvious (but are not necessarily easy to solve), the problems which come about as a result of the influence of the measurement scheme on the office itself are not usually so obvious. If a scheme is not carefully evaluated it can modify the office itself in undesirable ways. On the other hand, the influence of a measurement plan on the behavior of the office is potentially useful for inducing desired objectives. However, to attempt such inductions requires a good deal of knowledge about the offices.

A final, and perhaps tangential, difficulty with any analytic measurement scheme is that it will not itself separate the offices into those which are "efficient" and those which are "inefficient." Such a separation is usually achieved by a comparison with norms which may be obtained from statistical studies or from theoretical considerations. At some stage the separation always requires the judgment of management.

In summary, a measurement scheme, to be useful to management, must relate to and measure some understandable characteristics of office work performance in such a way that it is informative, and not potentially misleading. At the same time it must not interact with the actual office procedure in such a way that it invites the offices to become less efficient. It must allow the local managers to be flexible. It naturally follows that if meaningful office measurements can be constructed, they would be very helpful to both the immediate office management and the higher staff personnel.

This analysis was performed in conjunction with studies by the American Telephone and Telegraph Company and Bell Telephone Laboratories.

\section{THE DATA}

All analysis was carried out on an "entity" basis. The entities are groupings of office locations and "departments" such that each entity carries out approximately the same set of work functions. For example, some larger offices have part of the handling of telephone orders carried out by separate groups and not by the service representatives; these groups may even be at a different location but must be included in any interoffice comparisons. There are other similar situations and 
it was clearly necessary to construct groups as nearly alike in function as possible. While this grouping is necessary for consistent analysis, the details of it are not necessary for this paper. Finally, while the entities described above do not necessarily correspond to any other definition of a business office, they are referred to as offices in the remainder of this paper. No misunderstanding of this terminology should occur.

The basic data were of five different types: (i) daily counts of customer contacts, (ii) daily gross time data, (iii) daily work sampling observations, (iv) monthly numbers of accounts carried by the offices, $(v)$ profile survey variables. Each of these types of data played an important role in the development of the statistical models; however, the final models do not use work sampling. Let us take a closer look at these data types.

(i) Much of the work of the business offices is generated by the customer on the telephone; some personal contact occurs in public offices, but relatively little. Most of these customer contacts are counted and classified. Eight of the categories are orders, toll inquiries, other billing inquiries, and miscellaneous contacts, each for business and residence customers. These eight are among the most important classifications, and account for most of the office working time.

(ii) The daily gross time spent on all categories of work is available as a normal accounting item. This gross time is the total work time for which commercial employees in an office are paid. It includes: $(a)$ time spent for the previously mentioned eight classifications of customer contacts; (b) time spent in the company's public office; (c) time spent on treatment work; $(d)$ time spent on teller work; $(e)$ normally scheduled relief time and personal time; $(f)$ idle time; $(g)$ time spent on work classified as general activity; and $(h)$ time spent on miscellaneous activities. These categories are listed mainly for information and understanding. The bulk of the analysis is dependent only upon the availability of gross time data. Time does not have to be available in subcategories.

(iii) Time slice work sampling studies were carried out in 46 offices of the System in 1964. This study gave daily estimates of the total time spent on the various work categories including the eight categories mentioned in item $i$. In 42 of these offices, data were gathered for a 13 -week period from May through July, and in the remaining four categories, the study continued for seven months through November 1964 .

(iv) The number of accounts carried by each of the 46 offices was 
obtained for each month the office was in the study. The numbers used are totals of both business and residence accounts.

$(v)$ A profile survey was made of all offices in the System to determine basic characteristics about each office and its environment. Data on over 200 exogenous variables were obtained, 55 of which were studied in detail. Only those used in the models described in this paper are explicitly introduced.

III. THE DEVELOPMENT OF THE SINGLE OFFICE MODEL

To construct a first model for daily time expenditure in a single office, assume that time is used up partly as a result of direct customer demands, and partly by overhead time, see equation (1).

$$
\left.\left[\begin{array}{c}
\text { time spent on all } \\
\text { commercial office } \\
\text { work }
\end{array}\right]=\text { [overhead time }\right]+\left[\begin{array}{c}
\text { time required for } \\
\text { customer generated } \\
\text { demands }
\end{array}\right] \text {. }
$$

Next, suppose that the time required to carry out a single customer contact in the $j$ th work category is $a_{i}$, and that it is performed $F_{i j}$ times on day $i$. Then the total time spent that day on category $j$ is $F_{i j} a_{i}$ and the right bracket of the right side of equation (1) could be written as $\sum_{i=1}^{k} F_{i j} a_{j}$, where $k$ is the total number of work categories. Thus, if $a_{o}$ denotes overhead time, equation (1) can be written as

$$
T_{i}=a_{o}+\sum_{i=1}^{k} F_{i j} a_{i},
$$

where $T_{i}$ is the total time expenditure on day $i, i=1,2,3, \ldots, n$. Since it is doubtful that such exact relationships ever hold, the model given in equation. (2) needs to be modified. Only the specific modifications used in this paper are discussed. For a more general discussion see Ref. 1.

The first modification was a transformation of all observations to logarithms. This transformation was performed because plots of the estimated daily time on each of the eight categories (using data from the work sampling study) against the corresponding daily contact frequencies showed that the two were related approximately logarithmically. Consequently such a transformation could be expected to improve the statistical characteristics of the models.

The second major modification in the model formulation was the reduction in the number of categories. This came about because multicollinearities among the independent variables led to an extensive study to find which work categories were the best predictors of time. 
The result was that two categories, total number of business contacts and total number of residence contacts were found to be better statistical predictors of time than any other combination of single categories or groupings of categories. While these two modifications are important they are intermediate steps and so the details have not been presented.

Consequently, the model used for each single office had the functional form,

$$
T_{i}=\beta_{0} F_{1 i}^{\beta_{1}} F_{2 i}^{\beta_{3}}, \quad i=1,2, \cdots, n
$$

where $T_{i}$ is the gross time on commercial operation on day $i$, $F_{1 i}$ is the total daily number of business contacts, and $F_{2 i}$ is the total daily number of residence contacts.

At this point, $\log \beta_{o}$ is an estimate of overhead time in an additive model like equation (2), and $\beta_{1}$ and $\beta_{2}$ are estimates of the average time requirements on a $\log$ basis.

This model was applied to each of the 46 offices individually. The statistical details using the data from an individual office are presented in a paper which emphasizes the statistical development of this model. $^{2}$

\section{THE DEVELOPMENT OF THE MULTIOFFICE MODEL}

\subsection{Selection of an Appropriate Model}

The models used in Section III are of the general form $t=q\left(f_{1}, f_{2}\right.$, $\ldots, f_{k}$ ) which relates the demand put on an office and the time consumed by it. Possibly the most interesting use of these models is to give estimates of the time required by an office to carry out a given demand. load. Such an estimate could then be compared with the actual time used to produce an efficiency factor. A natural way to do this is as a ratio, $E=$ allowed time/actual time. These factors could be computed monthly to follow the progress of an office.

The comparison of different offices is not so simple, however. There are a number of possible approaches. One of these is to obtain a model fit and an efficiency score, $E$, for each of the offices for a specified month and then to compare the office $E$ scores. This is in effect fitting a model to all the offices in which each office is associated with an individual set of parameters $\left(\beta_{0}, \beta_{1}, \beta_{2}\right)$. But such a model has two major defects for use in interoffice efficiency comparisons.

The first is that the approach would be very cumbersome for use 
with such a large number of offices, but the second is the most devastating. It is that comparisons of two offices by use of such a model gives an inefficient operation a time allowance which is based on its own inefficient procedures. Similarly, an efficient office would be hurt in the comparison by being given only a time allowance based on its own efficient organization. This is clearly what is not wanted.

This defect suggests fitting a three parameter model to all offices. This would allow all offices the same standard overhead time and the same standard times for business and residence contacts. Such a model would be tractable and would eliminate the defect of allowing each office a standard time based on its own procedures.

However, the proposal of a three-parameter model makes it very clear that there really may be valid reasons why one office should have different time allowances from another. Consequently, we seem to stand between two models, one which allows every office the same overhead and average time allowances, and one which gives every office different allowances based on their individual performances.

What is clearly needed at this stage is a method and a model which gives offices a fair time allowance, based on the factors which actually influence the performance times. Operationally, this means relating the estimates of $\left(\beta_{0}, \beta_{1}, \beta_{2}\right)$ for each office to the exogenous variables which were measured in the profile survey.

\subsection{The Adjustment for Overhead Time}

It has been pointed out that the $\log \beta_{o}$ can be interpreted as measures of overhead time.* It has also been pointed out that it does not seem reasonable for interoffice comparisons to allow each office its own overhead time. There are two reasons for this. One is that such a procedure allows an inefficient office a time credit based on its own inefficient procedures. The other is that one would expect that a well-run large office might have more overhead time associated with it than a poorly-run small office. This means that a measure of office size must be introduced to scale these estimates of overhead time. The one selected was $A_{i}$, the monthly number of accounts carried by the office. Figure 1 is a plot of $\log \hat{\beta}_{o i}$ against $\log \left(A_{i} / 100\right)$. There is one point for each of the 46 offices. A linear regression model was fitted to these data. While the statistical details of the fit affect the decision to use $A_{i}$ as a scale variable, they only indirectly affect the final model, and consequently are not presented.

* Overhead time, as used here, means time for which no frequency count can sensibly be made. 


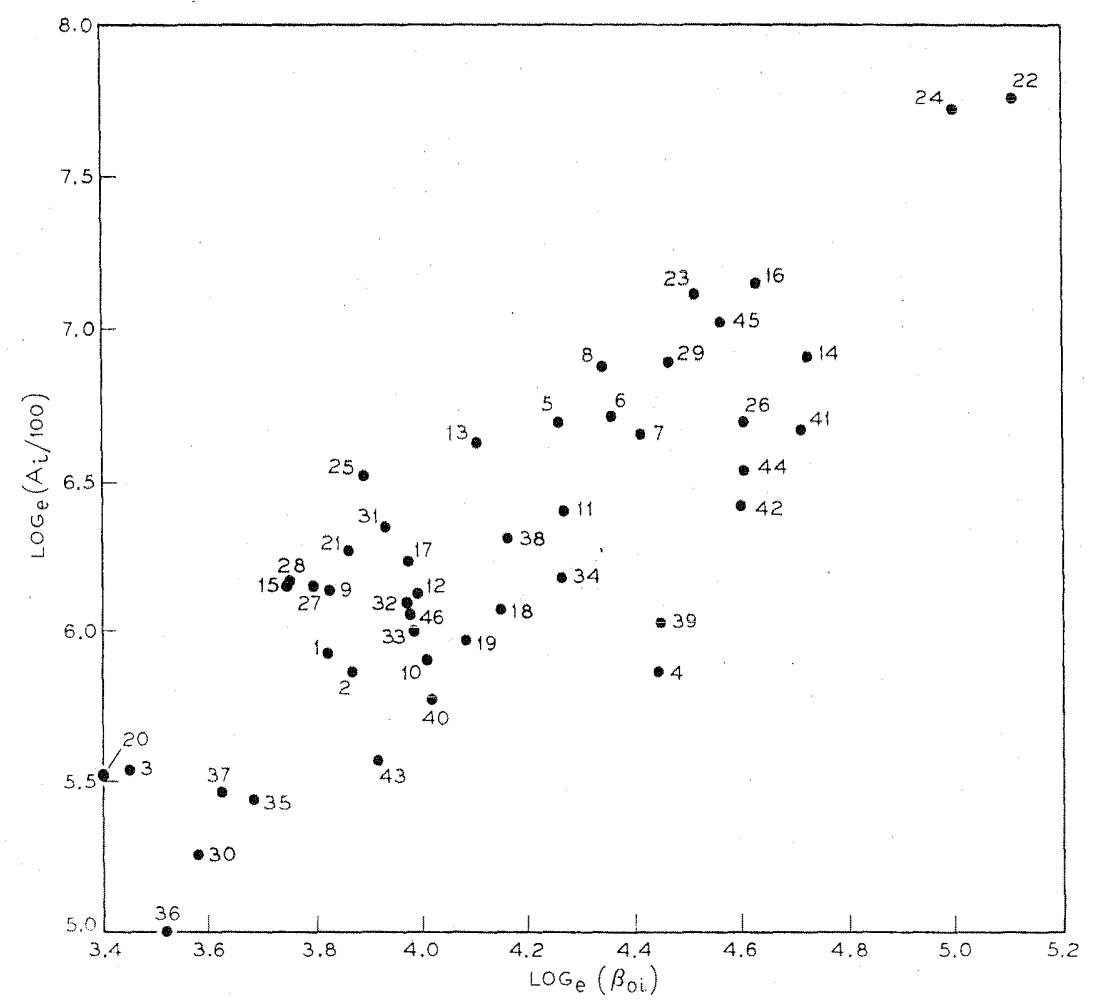

Fig. 1 - The number of office accounts versus the estimates of overhead time.

The good linear relationship between $\log \hat{\beta}_{o i}$ and $\log \left(A_{i} / 100\right)$ suggests modifying the model by inserting $\alpha_{0} A_{i}^{\alpha_{1}}$ for the $\hat{\beta}_{o i}$. This modification gives rise to equation (4),

$$
T_{i}=\alpha_{o} A_{i}^{\alpha_{1}} F_{1 i}^{\beta_{1}} F_{2 i}^{\beta_{2}}, \quad i=1,2, \cdots, 46
$$

where the parameters $\alpha_{0}, \alpha_{1}, \beta_{1}, \beta_{2}$ are common to all offices. Again the statistical details of the fit are not included.

\subsection{Adjustment for Contact Factors}

As has been pointed out, the time that it takes an office to carry out a business or residence contact may well be influenced by outside factors. The hope was that the estimates $\hat{\beta}_{1}$ and $\hat{\beta}_{2}$ of the business and residence contact times would be related to variables that were included among the profile variables. Consequently, a search of these variables was undertaken. 
Perhaps surprisingly, a number of good relationships were found. More, in fact, than were used. Percent service representative losses and number of main business telephones were found to be nicely related to the business parameter, $\beta_{1}$. Percent business main and number of customer bills handled were found to be usefully related to the residence parameter, $\beta_{2}$. The relationships were approximately logarithmic. Consequently, the multioffice model was modified in a manner similar to that for overhead time. Specifically, the multioffice model was put in the form,

$$
T=\alpha_{o} A^{\alpha_{1}} F_{1}^{\gamma_{0}+\gamma_{s} \log C_{2}} F_{2}^{\delta_{0}+\delta_{1} \log C_{s}},
$$

where $C_{1}$ and $C_{2}$ are the selected profile variables and the other variables, $T, F_{1}$ and $F_{2}$ remain as previously specified. In this form the time allowances (log basis), $\beta_{1}$ and $\beta_{2}$ have been modified so that each office's allowance is adjusted by the related profile variable.

So for example, if $C_{1}$ is percent service representative loss and $C_{2}$ is percent business main, the time allowance for an office would be made up of two components as originally specified.

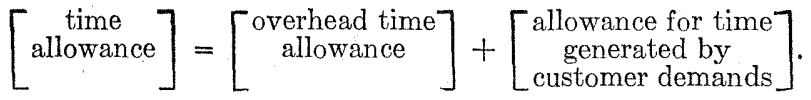

Now, however, the overhead time allowance is based on the size of the offices as specified by the number of accounts it carries. In addition, the time allowed for customer generated demands is based on the (log) number of contacts multiplied by an allowed time per contact. The time per business contact is bigger for offices with higher service representative losses. For residence, however, the time per contact is higher for offices which have a higher business main percentage. Apparently in these cases the residence customer requires more time to handle.

Percent service representative losses and percent business main telephones are not the only factors that can be used successfully. As stated earlier, a number of other variables are nicely related to the parameters $\beta_{1}$ and $\beta_{2}$ and have approximately the same statistical efficiency. In addition, the inclusion of even more exogeneous variables can reduce the residual mean square error of the fit. For example, in Ref. 2 the average time required for a residence contact is effectively related to both percent business main telephones and the total number of main stations. Then the model takes the form,

$$
T=\alpha_{0} A^{\alpha_{2}} F_{1}^{\gamma_{0}+\gamma_{2} \log C_{2}} F_{2}^{\delta_{0}+\delta_{2} \log C_{2}+\delta_{2} \log C_{2}} \text {, }
$$

where $C_{3}$ is the total number of main stations. 
Table I-Analysis of Variance (Log basis):

MOdified MOdeL

\begin{tabular}{c|c|r|r}
\hline Source & $\begin{array}{c}\text { Degrees of } \\
\text { Freedom }\end{array}$ & $\begin{array}{r}\text { Sum of Squares } \\
\text { (Fitted in order) }\end{array}$ & Mean Square \\
\hline $\log \alpha_{0}$ & 1 & $96,482.4814$ & $96,482.4814$ \\
\hline$\alpha_{1}$ & 1 & 781.4373 & 781.4373 \\
$\gamma_{0}$ & 1 & 111.4103 & 111.4103 \\
$\delta_{0}$ & 1 & 38.6524 & 38.6524 \\
$\gamma_{1}$ & 1 & 6.8262 & 6.8262 \\
$\delta_{1}$ & 1 & 24.7510 & 24.7510 \\
\hline Subtotal & & & \\
$\alpha_{1} \gamma_{0} \delta_{0} \gamma_{1} \delta_{1}$ & 5 & 963.0752 & 192.6150 \\
\hline Residual & 2952 & 98.4815 & 0.0333 \\
Total & 2958 & $97,544.0410$ & \\
\hline
\end{tabular}

For illustration, Table I gives the details of the analysis of variance for the model of equation (5), where $C_{1}$ is percent service representative loss and $C_{2}$ is percent business main telephones. Table II gives the estimates of the parameters from the complete fit, along with their standard errors. Table III presents the correlations among the estimated parameters. One important aspect of such correlations is that

Table II - Estimates of Parameters:

MODIFIEd MODEL

\begin{tabular}{c|c|c}
\hline Parameter & Estimate & Standard Error \\
\hline $\log \alpha_{0}$ & 0.1763 & 0.0040 \\
$\alpha_{1}$ & 0.4400 & 0.0131 \\
$\gamma_{0}$ & 0.1440 & 0.0074 \\
$\delta_{0}$ & 0.2935 & 0.0106 \\
$\gamma_{1}$ & 0.0413 & 0.0027 \\
$\delta_{1}$ & 0.2507 & 0.0092 \\
\hline
\end{tabular}

Table III-Correlation of Estimates of Parameters: Modrfied Model

\begin{tabular}{crrrrrr}
\hline & \multicolumn{1}{c}{$\log \alpha_{0}$} & \multicolumn{1}{c}{$\alpha_{1}$} & \multicolumn{1}{c}{$\gamma_{0}$} & \multicolumn{1}{c}{$\delta_{0}$} & \multicolumn{1}{c}{$\gamma_{1}$} & \multicolumn{1}{c}{$\delta_{1}$} \\
\cline { 2 - 7 } $\log \alpha_{0}$ & 1.000 & -0.622 & 0.386 & 0.027 & -0.035 & -0.370 \\
$\alpha_{1}$ & -0.622 & 1.000 & -0.458 & -0.724 & 0.128 & 0.400 \\
$\gamma_{0}$ & 0.386 & -0.458 & 1.000 & -0.090 & -0.038 & -0.663 \\
$\delta_{0}$ & 0.027 & -0.724 & -0.090 & 1.000 & -0.235 & -0.070 \\
$\gamma_{1}$ & -0.035 & 0.128 & -0.038 & -0.235 & 1.000 & 0.047 \\
$\delta_{1}$ & -0.370 & 0.400 & -0.663 & -0.070 & 0.047 & 1.000 \\
& & & & & & \\
\hline
\end{tabular}


the actual values obtained as estimates of the parameters cannot be separated from the model used to obtain them. For example, the estimate of $\delta_{1}$ is not the same in the models of equations (5) and (7).

Another feature of these exogeneous variables is that their selection and use in a measurement scheme will be heavily influenced by nonstatistical factors. The reason is that the mere selection of variables to be included in a measurement scheme can influence the operation of the office. If not carefullly selected the measured variables may become ends in themselves and the office may operate in such a way that its objective is not performing the real work function, but rather getting credit for the measurement scheme. Such a situation could even prevent office reorganization. An office may not feel inclined to automate if such a modernization would eliminate items for which credit is given. These are undesirable results; but it is also true that this type of an interaction can be used to bring about more favorable ends. For example, if larger offices are thought to be desirable, the allotment of larger time credits to larger offices would probably create a movement towards consolidation.

It is interesting to ask how the inclusion of percent service representative losses in a measurement scheme would affect the offices. One answer is that it seems unlikely that a manager would or could try to remove employees in order to increase the turnover rate. He already has considerable pressure on him to keep these losses as small as possible. However whether this is an accurate statement or not, this example makes it clear that major management decisions are needed during the development of any measurement plan.

In summary, it seems clear that the decision to include any variable in a measurement plan should be influenced not only by the statistical characteristics of the variable but also by very careful management, considerations.

\section{SOME ACTUAL OFFICE COMPARISONS}

The suggested measurement basis gives each office a time allotment based on the number of business and residence contacts handled and an adjusted (by the profile variables) standard time per contact, plus an allotment for overhead time based on the size (number of accounts) of the office. The formula is given in equation (8) using percent service representative losses and percent business main. This allotment is to be compared with the actual time consumed. Presumably this would be done each month.

It seems most natural to compare the allotted and actual times as 
a percentage; see equation (9). Other comparisons would be possible, such as one based on the difference of the actual and allotted times, but the percentage seems preferable because of its more natural scaling. The formula used is

$\left[\begin{array}{c}\text { alloted time } \\ \text { for office } i\end{array}\right]=1.193 A_{i}^{0.440} F_{1 i}^{0.144+0.041 C_{2}} F_{2 i}^{0.293+0.251 C_{2}}$

where $A_{i}$ is the monthly number of accounts carried by the office, $F_{1 i}$ is the daily number of business contacts,

$F_{2 i}$ is the daily number of residence contacts,

$C_{1}$ is the monthly percent Service Representative loss,

$C_{2}$ is the percent business main telephones for the month.

$$
E=\frac{\text { time allotment }}{\text { actual time reported }} \times 100 \text {. }
$$

Thus at the end of each month each office receives a rating which tells how it performed in relation to its own time standard. This allows two types of comparisons. The first is the month to month comparison of each office with itself; the second is the comparison of offices with each other on the basis of their percent efficiency. It is important to notice that these are different comparisons. It would not be impossible for an office to slip in comparison with itself from one month to the next but rank higher when compared with all other offices.

Based on the data of the three month study, the suggested procedure gives the rankings shown in Table IV. Notice that the rankings are relatively stable and that cases do occur in which the $E$ number and the ranking go in opposite directions from one month to the next. For example, consider offices 4 and 34 .

After these rankings were calculated, they were checked for obvious systematic behavior. None was found. The $E$ values are not related to the gross time used by the office nor to any of the variables used as inputs to the estimated time. This means that the scheme does not seem to be favoring offices with special characteristics.

VI. PRINCIPAL STEPS IN FORMING THE MODEL

The key steps which lead to the final model formulation are:

(i) The formation of the entities. This allows analysis of comparable office groupings without which consistent statistical relationships would probably not have been found.

(ii) The recognition that the relationship between time consumed and demand load is nonlinear and that a log transformation allows 
Table IV-Office $E$ Numbers and Rankings

\begin{tabular}{|c|c|c|c|c|c|c|}
\hline \multirow{2}{*}{$\begin{array}{c}\text { Office } \\
\text { Designation }\end{array}$} & \multicolumn{2}{|c|}{ Month 1} & \multicolumn{2}{|c|}{ Month 2} & \multicolumn{2}{|c|}{ Month 3} \\
\hline & Rank & $E$-Value & Rank & $E$ Value & Rank & $E$-Value \\
\hline 1 & 26 & 96.11 & 37 & 93.98 & 27 & 99.42 \\
\hline 2 & 41 & 83.52 & 34 & 94.69 & 32 & 95.40 \\
\hline 3 & 15 & 105.77 & 16 & 107.29 & 20 & 104.57 \\
\hline 4 & 18 & 98.87 & 20 & 102.67 & 36 & 92.44 \\
\hline 5 & 11 & 111.60 & 8 & 118.58 & 3 & 123.10 \\
\hline 6 & 34 & 93.18 & 21 & 102.63 & 14 & 108.40 \\
\hline 7 & 29 & 94.40 & 32 & 96.07 & 38 & 92.26 \\
\hline 8 & 20 & 98.41 & 22 & 102.06 & 21 & 103.27 \\
\hline 9 & 8 & 115.29 & 12 & 114.08 & 11 & 115.09 \\
\hline 10 & 27 & 95.57 & 39 & 92.94 & 41 & 85.66 \\
\hline 11 & 42 & 80.50 & 45 & 75.73 & 43 & 78.75 \\
\hline 12 & 39 & 89.58 & 23 & 101.13 & 22 & 101.21 \\
\hline 13 & 10 & 112.04 & 11 & 115.40 & 8 & 119.22 \\
\hline 14 & 46 & 70.79 & 46 & 74.32 & 45 & 73.34 \\
\hline 15 & 3 & 123.27 & 4 & 121.93 & 5 & 121.50 \\
\hline 16 & 19 & 98.61 & 24 & 99.38 & 24 & 100.47 \\
\hline 17 & 4 & 120.53 & 7 & 119.10 & 10 & 116.77 \\
\hline 18 & 33 & 93.25 & 35 & 94.46 & 31 & 95.78 \\
\hline 19 & 21 & 98.01 & 38 & 93.97 & 23 & 100.65 \\
\hline 20 & 7 & 116.60 & 5 & 120.60 & 6 & 120.83 \\
\hline 21 & 9 & 114.94 & 6 & 120.53 & 2 & 129.15 \\
\hline 22 & 36 & 91.23 & 36 & 94.07 & 34 & 93.67 \\
\hline 23 & 13 & 109.49 & 15 & 111.11 & 9 & 117.14 \\
\hline 24 & 23 & 97.31 & 26 & 99.15 & 33 & 94.12 \\
\hline 25 & 1 & 159.53 & 1 & 138.73 & 7 & 120.68 \\
\hline 26 & 5 & 118.60 & 13 & 111.73 & 16 & 107.15 \\
\hline 27 & 6 & 118.48 & 3 & 128.52 & 4 & 121.97 \\
\hline 28 & 2 & 130.53 & 2 & 130.76 & 1 & 132.68 \\
\hline 29 & 35 & 92.62 & 25 & 99.17 & 35 & 93.26 \\
\hline 30 & 38 & 89.81 & 30 & 96.48 & 29 & 98.34 \\
\hline 31 & 28 & 94.80 & 19 & 103.05 & 12 & 112.83 \\
\hline 32 & 14 & 109.22 & 10 & 116.47 & 19 & 106.14 \\
\hline 33 & 37 & 90.41 & 27 & 99.00 & 30 & 96.08 \\
\hline 34 & 31 & 93.69 & 33 & 94.94 & 17 & 106.60 \\
\hline 35 & 32 & 93.51 & 31 & 96.11 & 37 & 92.33 \\
\hline 36 & 22 & 98.00 & 40 & 91.44 & 40 & 86.92 \\
\hline 37 & 24 & 97.12 & 28 & 97.94 & 1.5 & 107.29 \\
\hline 38 & 25 & 96.12 & 17 & 105.94 & 25 & 99.95 \\
\hline 39 & 30 & 93.78 & 29 & 97.41 & 28 & 98.43 \\
\hline 40 & 17 & 102.32 & 14 & 111.26 & 26 & 99.92 \\
\hline 41 & 45 & 74.47 & 43 & 77.79 & 42 & 80.03 \\
\hline 42 & 43 & 79.23 & 41 & 81.59 & 39 & 90.83 \\
\hline 43 & 40 & 84.41 & 44 & 77.15 & 44 & 76.83 \\
\hline 44 & 44 & 75.79 & 42 & 78.32 & 46 & 72.17 \\
\hline 45 & 16 & 104.34 & 18 & 104.60 & 18 & 106.50 \\
\hline 46 & 12 & 110.14 & 9 & 116.77 & 13 & 112.13 \\
\hline
\end{tabular}


simple and effective fitting. This transformation also has the important advantage of stabilizing the variances, thus making the spread of the resulting $E$ estimates about the same for different classes of entities.

(iii) The recognition that the business-residence classification of customer contacts was more closely related to time usage than any other work categories. This grouping not only predicts time very well, but also requires substantially less data gathering than the more detailed classifications. In addition, since System offices tend to be organized according to the business-residence function, data gathering for this classification might possibly be completely automated.

(iv) The recognition that gross time can be predicted with more accuracy than the time associated with any subcategories. This means that work categories for which no frequency counts are available, are included in the analysis as "overhead" time. It also means that no work sampling is required.

$(v)$ The introduction of the number of accounts as a measure of office size and its use in scaling the estimates of overhead time. Similarly, the use of the profile variables for adjusting the average time made the office comparisons more equitable.

\section{SUMMARY}

This paper has described the development of statistical models for time usage in Bell System business offices. These experimental models have been designed so that they are good predictors of time, and can be used to give time allowances to different offices in an equitable way. The latter requirement means that suitable external variables have to be included. The manner in which this is carried out (see Section 4) is one of the key parts of the paper.

Finally it is pointed out that although work sampling may give very useful information in time studies, the use of a measurement scheme based on statistical models of the kind suggested in the paper would not require it. Data obtained by work sampling was used in the analysis but is not necessary for the general application of this approach.

\section{ACKNOWLEDGMENTS}

Many people have contributed significantly to this project. This is particularly true of persons in the Bell System operating companies. 
There are far too many of them to be named. All of the actual observations and preliminary data tabulations were made by these people and without them no results of any kind would ever have been achieved. In addition, many of them patiently took us on guided tours of their business offices and carefully explained their operation to us. This document owes all of them a great deal.

Claire Gerity and his group at the American Telephone and Telegraph Company were very instrumental and cooperative in making arrangements and passing along their insight into the business office operations. In particular, David Macarthy of that group must be singled out for his valuable contributions.

\section{REFERENCES}

1. Williams, W. H., "A Linear Model Approach to Time and Cost Analysis," Management Science, 12, No.6 (February 1966) pp. B216-223.

2. Williams, W. H., and Chen, H., unpublished work. 